\title{
Low alpha power (7.5-9.5 Hz) changes during positive and negative affective learning
}

\author{
D. ERIK EVERHART \\ East Carolina University, Greenville, North Carolina \\ and \\ HEATH A. DEMAREE \\ Case Western Reserve University, Cleveland, Ohio
}

\begin{abstract}
There is evidence that the positive and the negative word lists of the Affective Auditory Verbal Learning Test (AAVL) are useful with regard to mood induction. To date, however, changes in brain activation, as indicated by quantitative electroencephalographic recording, have not been examined. Thus, changes in low alpha power $(7.5-9.5 \mathrm{~Hz})$ were examined during and after completion of the positive or the negative learning list of the AAVL among 37 undergraduate men and women. Three primary findings from the study include the following: (1) Previously reported recall patterns were replicated; (2) participants who completed the negative list reported a significant decline in mood state at the end of the session; and (3) participants who completed the negative word list evidenced a significant reduction in low alpha power (in comparison with baseline) within the parietal regions. The findings noted above are seemingly counter to contemporary theories of mood regulation (i.e., asymmetrical changes in anterior activity, rather than changes in parietal regions). Although the AAVL may have limited utility as a tool for mood induction, it may serve as a functional tool for examination of the cerebral processes associated with affective verbal memory.
\end{abstract}

It has been suggested that the use of emotional stimuli such as the Affective Auditory Verbal Learning Test (AAVL; Snyder \& Harrison, 1997) in neurocognitive examination (i.e., memory) may provide fruitful information with regard to the relationship between cognitive and emotional functions (Borod, Tabert, Santschi, \& Strauss, 2000). Use of such measures may also be of benefit in the evaluation of individuals who suffer from an affective disorder (Mitrushina, Boone, \& D'Elia, 1999).

One measure that utilizes emotional stimuli, the AAVL, was initially developed as an alternative to the Rey Auditory Verbal Learning Test (RAVL; Rey, 1964) in order to provide an objective measure of affective verbal processing within the auditory modality. The AAVL consists of two (positive and negative) 15-item word lists that were developed using Toglia and Battig's (1978) list of word norms. Similar to the administration procedures of the RAVL, participants are required to learn the words across five successive trials. In the initial study, Snyder and Harrison (1997) examined differences in acquisition, primacy, and recency patterns for positive and negative word lists. Although positive and negative word lists yielded comparable acquisition scores (i.e., similar learning curves and number of words recalled), significantly dif-

Correspondence concerning this article should be addressed to D. E. Everhart, Department of Psychology and Program in Neuroscience, East Carolina University, Rawl Building, Greenville, NC 27858 (e-mail: everhartd@mail.ecu.edu). ferent primacy and recency effects were noted. Specifically, a greater primacy effect was noted among participants who were asked to learn the negative list than among participants who were asked to learn the positive list. In contrast, a greater recency effect was noted among participants who were required to learn the positive list, in comparison with the negative list. These findings suggest that the AAVL may be useful in the examination of the effects of valence (positive vs. negative) on learning.

There is also empirical evidence that the positive and the negative word lists of the AAVL are associated with differing patterns of physiological arousal following administration. In a replication of the experiment described above, Snyder, Harrison, and Shenal (1998) found increased diastolic blood pressure and mean arterial pressure following negative list learning and decreased diastolic blood pressure and heart rate following positive list learning. Also of note, the primacy and recency patterns found by Snyder and Harrison (1997) were replicated.

Taken together, these findings suggest that the AAVL may be useful in the examination of the effects of valence (positive vs. negative) on learning. Presently, however, the relationship between learning the positive and negative word lists from the AAVL and changes in brain activity is unknown. Understanding the changes in regional brain activity following encoding of emotional verbal material would be important, particularly if the AAVL is to be used for mood induction studies among clinical populations.

In the present study, quantitative electroencephalographic (QEEG) recording is used to examine changes in 
brain activity as a function of learning the positive or the negative word lists from the AAVL. Previous studies have found that QEEG recording is a sensitive measure of brain activity during presentation of verbal stimuli. Specifically, power spectral analysis of the EEG waveform during mental tasks yields a decrease in alpha band (8-12 Hz) power (Dolce \& Waldeier, 1974; Glass, 1966; Osaka, 1984). Of note, power within the alpha band is inversely correlated with relative brain activation; a decrease in alpha power is indicative of an increase in brain activation (Davidson, 1995; Davidson \& Henriques, 2000; Lindsley \& Wicke, 1974; Shagass, 1972). Alpha power attenuation has been observed during the encoding of verbal information (Klimesch, Doppelmayr, Russegger, \& Pachinger, 1996). There is also support that power within the alpha band decreases in the left hemisphere under verbal loads and decreases in the right hemisphere under nonverbal loads (Burgess \& Gruzelier, 1997; Butler \& Glass, 1974; Galin \& Ornstein, 1972).

With regard to emotion, EEG alpha power is also sensitive to changes in emotional state following induction and characterological differences in affective style. For instance, Davidson, Ekman, Saron, and Friesen (1990) found right anterior activation while adult participants viewed emotional films designed to induce negative emotion. In contrast, left anterior activation was observed during films that were designed to induce positive emotion. With respect to characterological differences in affective style, Schaffer, Davidson, and Saron (1983) found less frontal activation among depressed patients than among nondepressed controls. The same pattern was found among remitted depressives, as compared with controls (Henriques \& Davidson, 1990), indicating that such patterns may be characterological.

Therefore, the primary purpose of the present study was to further evaluate the utility of the AAVL with regard to emotion induction, using changes in alpha power as a dependent measure. Demonstrable changes in brain activation, as measured by QEEG, would be expected following presentation of positive versus negative words. If the AAVL is associated with mood induction, then (1) right frontal activation (F4, F8) would be expected following presentation of negative words, whereas (2) left frontal activation (F3, F7) would be expected following presentation of positive words. One shortcoming found in the previous studies (Snyder \& Harrison, 1997; Snyder et al., 1998) was the absence of self-reported changes in mood following presentation of the AAVL, due to the lack of a manipulation check. In the present study, participants were asked to rate mood state, using a Likert scale before and after presentation of AAVL stimuli. If the negative word list from the AAVL produces decline in mood state, then (3) participants would be expected to self-report changes in mood state, and (4) these changes would correlate with predictable shifts in frontal asymmetry (positive-left, negative-right).

Despite having hypotheses restricted to frontal EEG asymmetry, data from all 10-20 electrode sites were collected in order to maximally assess the impact of the
AAVL. The authors acknowledge that emotional verbal memory, independent of mood, may influence relatively caudal brain regions. Data concerning the processing of emotional verbal memory have been mixed, with some research supporting the right-hemisphere model (Cimino, Verfaellie, Bowers, \& Heilman, 1991; Master, Thompson, Dunn, \& Lishman, 1986) and other research supporting the valence model (Ali, 1999; Ali \& Cimino, 1997). Given their use of healthy subjects, Ali and Cimino's study is particularly pertinent to the present research. Using a tachistoscope paradigm, these investigators found that accuracy scores for positively valenced words were significantly better if the words were presented to the right visual field (left hemisphere). No such relationship was found for accuracy scores when negative words were presented to the left visual field (right hemisphere). The authors concluded that the failure to find significant associations between the negative words and the right hemisphere may be attributable to a basal effect that is associated with the verbal nature of the stimuli. However, in the recognition memory portion of the same paradigm, positive words presented to the left hemisphere were recognized with greater accuracy than were negative words. The opposite findings were observed for words presented to the right hemisphere; negative words were recognized with greater accuracy. Thus, the differences in caudal activity (and possibly caudal asymmetry) observed after learning positive or negative words may be attributable to affective memory processes.

\section{METHOD}

\section{Participants}

Right-handed male (8) and female (29) participants $(N=37)$ between the ages of 18 and 23 years were recruited from the departmental undergraduate participant pool. All the participants obtained extra credit toward their psychology course for participation. The research was approved by the University and Medical Center Review Board at East Carolina University.

\section{Word Lists}

Negative and positive affective word lists of the AAVL were developed using Toglia and Battig's (1978) index of word norms, which replicated and expanded the work of previous researchers (Locascio \& Ley, 1972). A total of 2,854 words were evaluated by 2,500 undergraduates at the University of Colorado, using a 7-point Likert scale for concreteness, imagery, categorizability, meaningfulness, familiarity (FAM), number of attributes, and pleasantness (PLS). Construction of the affective lists of the AAVL test consisted of selecting 15 words having the highest mean PLS rating and 15 words having the lowest mean PLS rating from the subset of all words with a FAM rating of at least 5.0 or greater (see Snyder \& Harrison, 1997; Snyder et al., 1998). The original RAVL list can also be referred to as a neutral list, since the PLS ratings $(M=4.38$, $S D=1.26$ ) for 11 of these words (4 words were not in the index) were near the middle (4.0 rating) of the 7-point Likert scale for PLS. Positive and negative word lists were tape recorded onto TDK IECI/Type I audio tapes at a rate of approximately $2 \mathrm{sec}$ per word and played at about $50 \mathrm{~dB}$ on a Sony CFD-G30 CD radio cassette player. A female actor was used for all voice recordings.

\section{QEEG Recording Procedures}

The participants were tested in the Cognitive Neuroscience Laboratory located within the Department of Psychology at East Car- 
olina University. The participants were seated in a reclining chair and were fitted with a lycra electrode cap (Electro-Cap International, Inc.). Within the electrode cap, electrodes are arranged according to the 10-20 international system (Jasper, 1958). Thus, EEG was recorded from 19 active electrode sites using linked ears (A1 and A2) as a reference (monopolar montage). The use of linked ears is recommended for asymmetry research as the best reference when using a specific, rather than a computed, reference location (Hagemann, Naumann, \& Thayer, 2001). In order to record eye movements, one electrode was placed at the outer canthus of the left eye (horizontal eye movements), and one electrode was placed supraorbitally above the left eye (vertical eye movements). Thus, eye movements were recorded on two separate auxiliary channels. The EEG signal was amplified and converted on line to digital, using a Lexicor Neurosearch - 24 Brain Mapping System. The amplification factor was 32,000 , with a sampling rate of 256 samples/sec. A high-pass filter was used to eliminate slow wave frequencies that were less than $2 \mathrm{~Hz}$. A $60-\mathrm{Hz}$ notch filter was used to eliminate $60-\mathrm{Hz}$ line noise. Data were stored and analyzed on a PC Pentium computer. The EEG data were converted on line for display, storage, and analysis. The participants were instructed to close their eyes for all recordings. In total, four 1-min EEG samples were recorded for each participant (baseline, immediately after learning Trial 3 of the AAVL, immediately after Trial 5 of the AAVL, and 5 min after completion of the AAVL). Artifacting was completed using a two-step process. First, epochs that contained signals that were greater than $\pm 50 \mu \mathrm{V}$ were automatically eliminated via computer software. Second, on the basis of visual inspection of auxiliary channels, epochs that contained significant eye movements were eliminated. In all cases, at least $85 \%$ (i.e., 51 one-second epochs) of each of the 1-min recordings remained for fast Fourier transformation (FFT). Power spectral analysis (using FFT) was subsequently performed on 1-sec epochs; the data for each 1-sec epoch were averaged together for each of the four 1-min recording periods.

\section{Procedures}

Upon arrival, the participants were asked to read and sign an informed consent form. A brief medical history survey was used to eliminate participants with a history of head injury, drug use (e.g., alcohol, prescription drugs, etc.), psychological problems (e.g., depression, attention deficit disorder, etc.), and diagnosis of learning disabilities. The participants were also asked to complete the behavioral laterality inventory (Coren, Porac, \& Duncan, 1979) in order to ensure right-hand dominance. A score of +6 on the inventory was required for further participation. Upon completion of questionnaires, the electrode application procedures described above were initiated. Following preparation and after a $10-\mathrm{min}$ adaptation period, the participants were asked to provide a numerical rating of overall mood state, using a Likert scale (presented visually), where +10 was considered an extremely positive mood state and -10 was considered an extremely negative mood state. Immediately after response, the experimenter left the room, and baseline EEG recording was initiated for the duration of $1 \mathrm{~min}$. Depending on arrival, a positive or a negative word list was read to the participants, using a previously recorded audiotape of a female voice reading the lists at about one word per second. Instructions were also read by the female voice on the audiotape, which was played through the intercom system at about $50 \mathrm{~dB}$. The participants received the following instructions from the original RAVL on Trial 1: "I am going to read you a list of words. Please listen carefully. When I stop, you are to say back as many words as you can remember. Say the words in any order you remember. Just try to remember as many as you can." Instructions for Trial 2 through Trial 5 were also as follows: "Now I'm going to read the same list again. When I stop again, I want you to tell me as many words as you can remember, including words you said the first time. It doesn't matter what order you say them. Just say as many words as you can remember whether or not you said them before." When the participant could no longer recall any more words or a maximum of $3 \mathrm{~min}$ had passed, the next trial began. The participant's responses were recorded on the data sheet. Following completion of the fifth learning trial, the participants were again asked to provide a Likert scale rating of mood state, using the same visual scale as that described above.

The experimental procedures were identical to those described by Snyder and Harrison (1997), with the exception of EEG recording. In total, four 1-min samples were recorded for each participant. A 1-min baseline recording (baseline) was initiated following a 10-min adaptation period and prior to initiation of the AAVL. Oneminute samples were also recorded immediately after the third trial of the AAVL, immediately after the final trial of the AAVL (Trial 5), and 5 min after completion of the AAVL (recovery). After the final EEG recording, the participants were debriefed and excused.

\section{RESULTS}

\section{Behavioral Data: Learning/Recall Patterns}

Independent analyses of variance (ANOVAs) were performed on three dependent memory variables: primacy, recency, and overall memory data. Each ANOVA was performed with an independent factor of valence (positive and negative word lists from the AAVL) and a repeated measure of trial (1-5). All pairwise comparisons were made using Tukey's honestly significant difference test to control for experimentwise error ratio (Winer, 1971).

For primacy data, analysis revealed a significant main effect of valence $[F(1,35)=23.10, p<.001]$. Fewer primacy words were recalled from the positive version of the AAVL $(M=3.69, S D=0.71)$ than from the negative $(M=4.59, S D=0.36)$. A significant main effect of trial was also found $[F(4,140)=17.34, p<.001]$. Fewer primacy words were recalled during Trial $1(M=2.68, S D=$ 1.16) than during Trial $2(M=3.63, S D=1.26)$, Trial 3 $(M=3.84, S D=0.96)$, Trial $4(M=3.95, S D=0.78)$, and Trial $5(M=4.37, S D=0.68)$. Moreover, fewer words were recalled during Trials 2,3 , and 4 relative to Trial 5. A significant valence $\times$ trial interaction was also found for primacy data $[F(4,140)=2.70, p<.05]$. Relative to negative words, fewer positive AAVL words were recalled during Trials $1,3,4$, and 5, but not during Trial 2.

For recency data, analysis revealed a significant main effect of valence $[F(1,35)=15.98, p<.001]$. Specifically, fewer primacy words were recalled from the negative version of the AAVL $(M=3.10, S D=0.68)$ than from the positive $(M=3.87, S D=0.52)$. A significant main effect of trial was also found $[F(4,140)=25.6, p<$ $.001]$. Specifically, more recency words were recalled during Trial $3(M=3.81, S D=1.05)$, Trial $4(M=4.00$, $S D=0.94)$, and Trial $5(M=4.11, S D=0.94)$ than during Trial $1(M=2.43, S D=1.30)$ and Trial $2(M=3.08$, $S D=1.01)$.

For total recall data, analysis revealed a significant main effect of trial $[F(4,140)=107.4, p<.001]$. The number of words recalled increased significantly from Trial $1(M=7.5, S D=1.69)$ to Trial $2(M=9.86, S D=$ 
$1.95)$ and from Trial 2 to Trial $3(M=11.73, S D=1.97)$. The number of words recalled also increased significantly from Trial $4(M=12.14, S D=1.95)$ to Trial 5 $(M=12.69, S D=1.76)$.

\section{Behavioral Data: Self-Report of Mood State}

Pretest and posttest ratings on mood state, using the Likert scale previously described, were recorded. The ratings were converted into one score (pretest - posttest) for comparison purposes. A one-way ANOVA yielded a significant difference between the positive and the negative word lists $[F(1,35)=5.25, p<.05]$. As was hypothesized, the participants who received the negative word list $(M=1.56, S D=1.88)$ reported a greater decline in mood state (i.e., toward the negative direction) than did the participants who received the positive word list $(M=0.37, S D=1.21)$. Thus, mood state is affected differently by the two word lists, in that mood is significantly altered with the negative list only.

\section{Data Reduction and QEEG Analyses}

Analyses were restricted to frequencies within the alpha band $(8-13 \mathrm{~Hz})$ and to the following electrode sites: F3-F4, T3-T4, T5-T6, C3-C4, P3-P4, and O1-O2. Initially, the alpha band was separated into three components including low alpha $(7.5-9.5 \mathrm{~Hz})$, mid alpha $(9.5-11.5 \mathrm{~Hz})$, and high alpha $(11.5-13.0 \mathrm{~Hz})$, as has been recommended by Crawford, Clarke, and Kitner-Triolo (1996). Next, three separate percentage of change scores were calculated for each electrode site, using absolute power spectrum values within each identified alpha band. This procedure was performed in order to reduce the amount of comparisons submitted for statistical analyses. Thus, the EEG sample recorded following Trial 3 of the AAVL was subtracted from the baseline recording for each site. Likewise, the EEG sample recorded following Trial 5 of the AAVL was subtracted from the baseline recording, and the EEG sample recorded 5 min (recovery) following completion of the AAVL was subtracted from the baseline recording. Next, three independent ANOVAs were performed (one for each component), with the between-subjects factor of valence (positive or negative) and the repeated factors of hemisphere (left and right), site (F3-F4, F7-F8, T3-T4, C3-C4, P3-P4, and O1-O2), and trial (Trial 3, Trial 5, and recovery). Initial analyses found no interactions or main effects involving valence within the mid and high alpha bands. These frequency bands were no longer considered for further analyses.

For the low alpha band, a significant valence $\times$ hemisphere $\times$ site $\times$ trial interaction was evidenced $[F(12,420)=$ $1.78, p<.05]$. Likewise, a significant valence $\times$ site $\times$ trial interaction was evidenced $[F(12,420)=2.13, p<$ $.01]$. No other interactions or main effects were present. The significant interactions were further explored using separate ANOVAs for each percentage of change score. Thus, a repeated measures design with the betweensubjects factor of valence (positive and negative) and the within-subjects factors of hemisphere (left and right) and site (F3-F4, F7-F8, T3-T4, C3-C4, P3-P4, and $\mathrm{O} 1-\mathrm{O} 2$ ) was performed for each of the three percentage of change scores separately. These analyses yielded a significant valence $\times$ site interaction $[F(6,204)=2.56, p<$ $.05]$ for the second percentage of change score only; no interactions or main effects were observed for the first or third percentage of change scores. Thus, the percentage of change in low alpha power differed between the two groups (positive and negative) only following completion of the AAVL. This difference is noted in Figure 1.

The valence $X$ site interaction noted for the second percentage of change score (i.e., immediately after completion of the AAVL) was further explored using planned post hoc comparisons. As can be seen in Table 1, the participants who received the negative word list evidenced lower alpha power within the parietal (P3-P4) scalp sites $[t(34)=2.07, p<.05]$. Nonsignificant trends were observed for the central $(\mathrm{C} 3-\mathrm{C} 4)$ scalp sites $[t(34)=1.69$, $p<.10]$ and the occipital $(\mathrm{O} 1-\mathrm{O} 2)$ scalp sites $[t(34)=$ $1.65, p<.10]$.

To help determine whether mood changes from the AAVL may have any influence on frontal asymmetry, separate Pearson product-moment correlations were performed between self-reported mood changes and low alpha power changes within frontal electrode sites (F3, F4, F7, and F8) for each group (i.e., positive vs. negative word list). Pearson product-moment correlations were also performed between self-reported mood changes and the remaining scalp sites (temporal [T3, T4, T7, T8], central [C3, C4], parietal [P3, P4], and occipital [O1, $\mathrm{O} 2]$ ). No significant correlations were revealed.

\section{DISCUSSION}

The primary purpose of this study was to further evaluate the utility of the AAVL as an effective mood induction instrument. Three major findings are reported from this study. First, with regard to acquisition patterns, the results of the present study are commensurate with previous findings (Snyder \& Harrison, 1997; Snyder et al., 1998). Specifically, the participants who were asked to learn a negative word list demonstrated a relatively greater primacy effect, in comparison with the participants who were asked to learn a positive word list. In contrast, the participants who were asked to learn the positive word list evidenced a relatively greater recency effect than did the participants who learned the negative word list. Interestingly, the mean number of words recalled across trials did not differ between the positive and the negative word lists. The findings from this study, as well as those from others (Demaree \& Everhart, in press; Demaree, Shenal, \& Everhart, 2003), represent replication from separate laboratories.

The present study differs from previous studies in that a self-report Likert scale measure of change in mood state was completed pre- and post-administration of the AAVL. The purpose of including this measure was to ensure that the AAVL was effective in inducing mood 


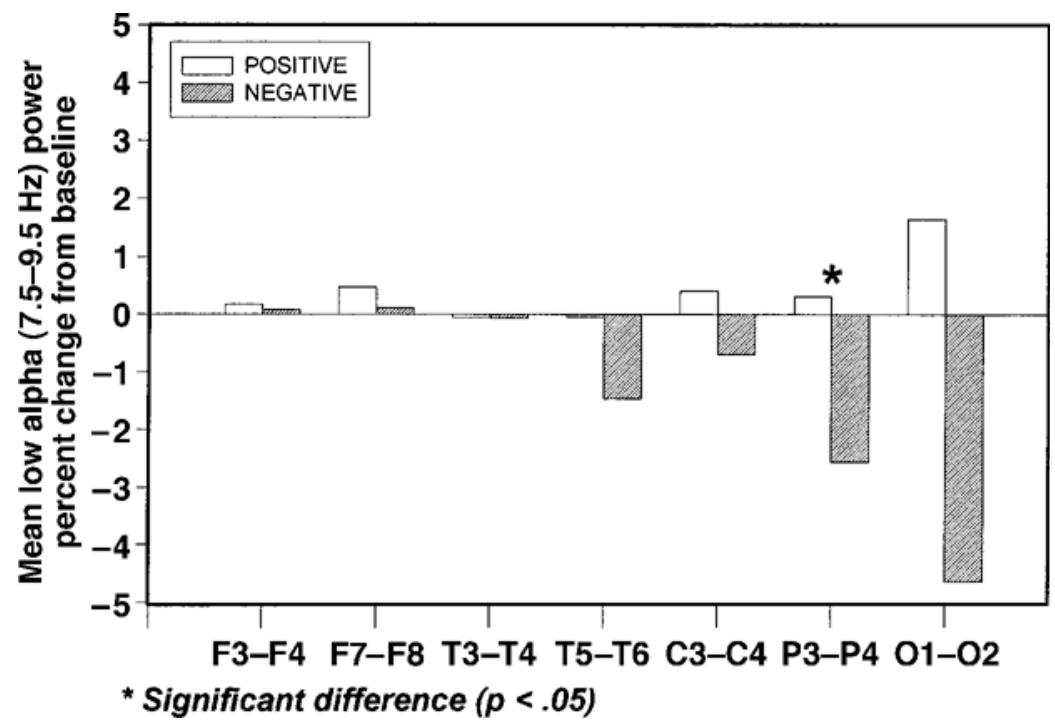

Figure 1. Mean percentage of change in low alpha (7.5-9.5 Hz) power in comparison with baseline immediately following completion of the AAVL. F3-F4, mid frontal; F7-F8, inferior frontal; T3-T4, anterior temporal; T5-T6, posterior tem poral; C3-C4, central; P3-P4, parietal; O1-O2, occipital. Decline in alpha power is inversely correlated with brain activation.

change (i.e., a manipulation check). The participants who learned the negative word list reported a modest decline in mood state following administration of the AAVL. In contrast, the participants who learned the positive word list reported no significant change in mood state. This finding suggests that the positive word list may not be effective in inducing positive changes in mood and that the AAVL may be limited as a mood induction procedure in this regard. In contrast, the significant findings with the negative word list suggest that the AAVL may be an effective negative mood induction procedure. One should be cautious when interpreting these results, however, since the experiment's demand characteristics may have influenced results toward significance.

Regarding the QEEG data, significant differences between positive and negative list learners were evidenced for low alpha $(7.5-9.5 \mathrm{~Hz})$, but not for mid alpha (9.5$11.5 \mathrm{~Hz})$ or high alpha $(11.5-13.0 \mathrm{~Hz})$. This finding is consistent with Crawford et al. (1996), where only the low alpha band successfully differentiated between positive and negative mood states. Also consistent with the present results is the previous finding that individuals who self-report poor sustained attentional abilities generate significantly more low alpha (i.e., decreased cortical activity) spectral magnitude during the performance of tracking or decision-making tasks (Crawford, Knebel, Vendemia, Kaplan, \& Ratcliff, 1995; Crawford \& Vasilescu, 1995).

It was specifically hypothesized that left frontal activation would be evidenced following completion of the positive list of the AAVL, whereas right frontal activation would be evidenced following completion of the negative list from the AAVL. These hypotheses stem from previous findings of changes in brain activation following presentation of emotional stimuli (for a review, see Davidson \& Henriques, 2000). This study did not find evidence of left frontal activation among the participants who learned the positive word list. Moreover, significant changes in alpha activity among positive list learners were not demonstrated across any scalp sites, regardless of hemisphere. It is possible that the lack of significant EEG findings results from the relative inability of the positive AAVL to induce an altered mood state.

It was also hypothesized that right frontal activation would be observed following completion of the negative list of the AAVL. As with the positive list, evidence for lateralized changes in brain activation was not demonstrated. However, significant bilateral differences in ac-

Table 1

Mean Percentage of Change (in Comparison With Baseline) in Low Alpha (7.5-9.5 Hz) Power Following Completion of the AAVL

\begin{tabular}{|c|c|c|c|c|c|c|}
\hline \multirow[b]{2}{*}{ Site } & \multicolumn{2}{|c|}{ Positive } & \multicolumn{2}{|c|}{ Negative } & \multirow[b]{2}{*}{$t$} & \multirow[b]{2}{*}{$p$ Value } \\
\hline & $M$ & $S D$ & $M$ & $S D$ & & \\
\hline F3-F4 & 0.18 & 0.85 & 0.08 & 0.97 & 0.31 & .76 \\
\hline F7-F8 & 0.48 & 1.49 & 0.11 & 1.60 & 0.72 & .48 \\
\hline $\mathrm{T} 3-\mathrm{T} 4$ & -0.58 & 0.64 & 0.39 & 0.54 & -0.62 & .54 \\
\hline T5-T6 & -0.50 & 2.10 & -1.46 & 4.18 & 1.31 & .20 \\
\hline $\mathrm{C} 3-\mathrm{C} 4$ & 0.41 & 1.37 & -0.70 & 2.40 & 1.70 & $.09 * *$ \\
\hline P3-P4 & 0.31 & 3.02 & -2.57 & 5.07 & 2.07 & $.04 *$ \\
\hline $\mathrm{O} 1-\mathrm{O} 2$ & 1.64 & 4.90 & -4.65 & 15.37 & 1.66 & $.10 * *$ \\
\hline
\end{tabular}

$* p<.05 . \quad * * p<.10$. 
tivation were observed between the participants who completed the negative versus the positive portion of the AAVL. Specifically, and as can be observed in Figure 1, the participants who completed the negative word list evidenced a greater percentage of decline in low alpha power (in comparison with baseline) when compared with the participants who completed the positive word list. Decrements in alpha power are thought to be inversely correlated with brain activation, and thus, there is greater brain activation within the specified regions (Davidson, 1995; Davidson \& Henriques, 2000; Lindsley \& Wicke, 1974; Shagass, 1972). It must be noted that this observed difference was noted only for the parietal electrode sites, although trends are indicated for other posterior sites (T5-T6, C3-C4, and $\mathrm{O} 1-\mathrm{O} 2$ ). Changes in mood have generally not been found to influence caudal arousal (but see Crawford et al., 1995), and the present data suggest that posterior asymmetry is not impacted by self-reported mood, as is implied by the nonsignificant correlations between changes in mood and parietal low alpha power. Rather, consistent with Ali and Cimino's (1997) research, the noted changes in posterior activity may reflect emotional list learning. More specifically, the findings (including changes in parietal regions noted during negative affective learning and the modest effects that the AAVL had on self-reported mood) suggest that the AAVL may be more useful in the study of emotional encoding and recall. Of note, although changes were observed within the parietal regions, no patterns of asymmetry were noted. The precise reason for this pattern of results is unclear, although such results are possibly attributable to the basal effect of the verbal nature of the stimuli, as was specified by Ali and Cimino. Relatedly, Grunwald et al. (1999) suggested that studies in which the perception of emotional words has been examined (i.e., lateralized paradigms, such as tachistoscopic techniques) have been less conclusive with regard to hemispheric specialization for emotion than have paradigms in which facial emotion or emotional prosody has been examined (for a review, see Borod, Bloom, \& Haywood, 1998). The reason(s) for the inconclusiveness are poorly understood, although they may be attributable in part to similar weaknesses in experimental design, as described below.

There were several weaknesses to the present study. First, there was lack of control with regard to the individual learning strategies of the participants. For example, some participants may have used self-verbalization to assist with encoding, whereas others may have used imagery. Second, changes in mood were assessed using a dimensional approach (i.e., Likert scale) rather than a categorical one. It has been well established that valence represents only one aspect of emotion and that negative and positive moods may not be orthogonally related $(\mathrm{Ca}-$ cioppo \& Berntson, 1994). In future studies, changes of discrete emotions should be assessed in order to better appraise the impact of affective stimuli on cerebral arousal. Moreover, it is plausible that, if the negative word list of the AAVL does induce negative mood as indicated by the participants, a complex interaction exists between mood state and encoding strategy. The design of the present study did not permit examination of such possible effects. Third, the AAVL word lists did not control for arousal level, a construct that may independently impact cerebral asymmetry (Heller, 1993). Thus, more extensive studies are required to ascertain the various factors involved in the encoding, retrieval, and recognition of affective words. Future studies may address the impact of the factors described above on the encoding and retrieval of the emotional words presented in the AAVL.

In summary, the findings of this study represent a replication and extension of prior research within a separate neuropsychological laboratory. The significant finding of self-reported decline in mood state following completion of the negative word list from the AAVL suggests that this procedure may be a valid instrument for negative mood induction (although presently, the findings from the study should be interpreted cautiously, due to the possible presence of demand characteristics), whereas the utility of the positive word list for mood induction may be more limited. The significant differences noted in low alpha power suggest that differences in brain activation do occur following administration of the negative versus the positive word list and that these differences may be limited to parietal regions. It is possible that the changes noted in cerebral activity do not stem from changes in mood; rather, they may be reflective of the processes required during emotional verbal memory.

\section{REFERENCES}

ALI, N. M. (1999). Hemispheric lateralization of memory for verbal emotional stimuli: Accounting for valence and arousal dimensions. Dissertation Abstracts International, 59(11-B), 6112.

Ali, N. [M.], \& Cimino, C. R. (1997). Hemispheric lateralization of perception and memory for emotional verbal stimuli in normal individuals. Neuropsychology, 11, 114-125.

Borod, J. C., Bloom, R., \& Haywood, C. S. (1998). Verbal aspects of emotional communication. In M. Beeman \& C. Chiarello (Eds.), Right hemisphere language comprehension: Perspectives from cognitive neuroscience (pp. 285-307). Mahwah, NJ: Erlbaum.

Borod, J. C., Tabert, M. H., Santschi, C., \& Strauss, E. H. (2000). Neuropsychological assessment of emotional processing in braindamaged patients. In J. C. Borod (Ed.), The neuropsychology of emotion: Series in affective science (pp. 80-105). New York: Oxford University Press.

Burgess, A. P., \& Gruzelier, J. H. (1997). Localization of word and face recognition memory using topographical EEG. Psychophysiology, 34, 7-16.

Butler, S. R., \& Glass, A. (1974). Asymmetries in the electroencephalogram associated with cerebral dominance. Electroencephalography \& Clinical Neurophysiology, 36, 481-491.

Cacioppo, J. T., \& Berntson, G. G. (1994). Relationship between attitudes and evaluative space: A critical review, with emphasis on the separability of positive and negative substrates. Psychological Bulletin, 115, 401-423.

Cimino, C. R., Verfaellie, M., Bowers, D., \& Heilman, K. M. (1991). Autobiographical memory: Influence of right hemisphere damage on emotionality and specificity. Brain \& Cognition, 15, 106-118.

Coren, S. P., Porac, C., \& Duncan, P. (1979). A behaviorally validated self-report inventory to assess 4 types of lateral preferences. Journal of Clinical Neuropsychology, 1, 55-64. 
Crawford, H. J., Clarke, S. W., \& Kitner-Triolo, M. (1996). Selfgenerated happy and sad emotions in low and highly hypnotizable persons during waking and hypnosis: Laterality and regional EEG activity differences. International Journal of Psychophysiology, 24, 239-266.

Crawford, H. J., Knebel, T. F., Vendemia, J. M. C., Kaplan, L., \& RATCLIFF, B. (1995). EEG activation patterns during tracking and decision-making tasks: Differences between low and high sustained attention adults. In Proceedings of the Eighth International Symposium on Aviation Psychology (pp. 886-890).

Crawford, H. J., \& VAsilescu, P. (1995). Differential EEG pattern activity of low and high sustained attention adults during decision-making tasks. Psychophysiology, 32, S26.

DAVIDSON, R. J. (1995). Cerebral asymmetry, emotion, and affective style. In R. J. Davidson \& K. Hugdahl (Eds.), Brain asymmetry (pp. 361-387). Cambridge, MA: MIT Press.

Davidson, R. J., Ekman, P., Saron, C. D., \& Friesen, W. V. (1990). Approach-withdrawal and cerebral asymmetry: Emotional expression and brain physiology I. Journal of Personality \& Social Psychology, 58, 330-341.

Davidson, R. J., \& Henriques, J. (2000). Regional brain function in sadness and depression. In J. C. Borod (Ed.), The neuropsychologyof emotion: Series in affective science (pp. 269-297). New York: Oxford University Press.

Demaree, H. A., \& Everhart, D. E. (in press). Healthy high-hostiles: Reduced parasympathetic activity and decreased sympathovagal flexibility during emotional processing. Personality \& Individual Differences.

Demaree, H. A., Shenal, B. V., \& Everhart, D. E. (2003). Primacy and recency effects found using affective word list. Manuscript submitted for publication.

Dolce, G., \& WALdeIer, H. (1974). Spectral and multivariate analysis of EEG changes during mental activity in man. Electroencephalography \& Clinical Neurophysiology, 36, 577-584.

Galin, D., \& ORnSTEIn, R. (1972). Lateral specialization of cognitive mode: An EEG study. Psychophysiology, 9, 412-418.

GLAss, A. (1966). Comparison of the effect of hard and easy arithmetic upon blocking of the occipital alpha rhythm. Quarterly Journal of Experimental Psychology, 18, 142-152.

Grunwald, I. S., Borod, J. C., Obler, L. K., Erhan, H. M., Pick, L. H., Welkowitz, J., Madigan, N. K., Sliwinski, M., \& Whalen, J. (1999). The effects of age and gender on the perception of lexical emotion. Applied Neuropsychology, 6, 226-238.

Hagemann, D., Naumann, E., \& Thayer, J. F. (2001). The quest for EEG reference revisited: A glance from brain asymmetry research. Psychophysiology, 38, 847-857.

Heller, W. (1993). Neuropsychological mechanisms of individual differences in emotion, personality, and arousal. Neuropsychology, 7, 476-489.
Henriques, J. B., \& DAvidson, R. J. (1990). Regional brain electrical asymmetries discriminate between previously depressed and healthy control subjects. Journal of Abnormal Psychology, 100, 535-545.

JASPER, H. H. (1958). The 10-20 electrode system of the international federaton. Electroencephalography \& Clinical Neurophysiology, 10, 317-375.

Klimesch, W., Doppelmayr, M., Russegger, H., \& Pachinger, T. (1996). Theta band power in the human scalp EEG and the encoding of new information. NeuroReport, 7, 1235-1240.

LindSLEY, D. B., \& WiCKE, J. D. (1974). The electroencephalogram: Autonomous electrical activity in man and animals. In R. Thompson \& M. N. Patterson (Eds.), Bioelectric recording techniques (pp. 3-83). New York: Academic Press.

LocAscio, D., \& LEY, R. (1972). Scaled-rated meaningfulness of 319 CVCVC words and paralogs previously assessed for associative reaction time. Journal of Verbal Learning \& Behavior, 11, $243-$ 250.

Master, D. R. Thompson, C., Dunn, G., \& Lishman, W. A. (1986). Memory selectivity and unilateral cerebral dysfunction. Psychological Medicine, 16, 781-788.

Mitrushina, M. M., Boone, K. B., \& D'Elia, L. F. (1999). Handbook of normative data for neuropsychological assessment. New York: Oxford University Press.

OsaKa, M. (1984). Peak alpha frequency of EEG during a mental task: Task difficulty and hemispheric differences. Psychophysiology, 21, 101-105.

REY, A. (1964). L'examen clinique en psychologie [The clinical psychological examination]. Paris: Universitaire de France.

Schaffer, C. E., Davidson, R. J., \& Saron, C. (1983). Frontal and parietal electroencephalogram asymmetry in depressed and nondepressed subjects. Biological Psychiatry, 18, 753-762.

SHAGASS, C. (1972). Electrical activity of the brain. In N. S. Greenfield \& R. A. Sternbach (Eds.), Handbook of psychophysiology (pp. 263328). New York: Holt, Rinehart \& Winston.

SNyder, K. A., \& HARrison, D. W. (1997). The affective auditory verbal learning test. Archives of Clinical Neuropsychology, 12, 477482.

Snyder, K. A., Harrison, D. W., \& Shenal, B. V. (1998). The affective auditory verbal learning test: Peripheral arousal correlates. Archives of Clinical Neuropsychology, 13, 251-258.

Toglia, M. P., \& BatTig, W. F. (1978). Handbook of word norms. Hillsdale, NJ: Erlbaum.

Winer, B. J. (1971). Statistical principles in experimental design (2nd ed.). New York: McGraw-Hill.

(Manuscript received March 7, 2002;

revision accepted for publication November 12, 2002.) 